\title{
Walk Your Talk: Real-World Adherence to Guidelines on the Use of MRI in Multiple Sclerosis
}

\author{
Mario Tortora ${ }^{1,+}{ }^{\dagger}$, Mario Tranfa ${ }^{1,+}{ }^{+}$, Anna Chiara D'Elia ${ }^{1}$, Giuseppe Pontillo ${ }^{1}{ }^{\mathbb{D}}$, Maria Petracca ${ }^{2,3}$, \\ Alessandro Bozzao ${ }^{4}{ }^{(\mathbb{D}}$, Ferdinando Caranci ${ }^{5}$, Amedeo Cervo ${ }^{6}$, Mirco Cosottini ${ }^{7}$, Andrea Falini ${ }^{8}$, \\ Marcello Longo ${ }^{9}$, Renzo Manara ${ }^{10}{ }^{\circ}$, Mario Muto ${ }^{11}$, Michele Porcu ${ }^{12}{ }^{(0)}$, Luca Roccatagliata ${ }^{13,14}$, \\ Alessandra Todeschini ${ }^{15}$, Luca Saba ${ }^{12} \odot$, Arturo Brunetti ${ }^{1}$, Sirio Cocozza ${ }^{1, *}$ and Andrea Elefante ${ }^{1}$
}

1 Department of Advanced Biomedical Sciences, University of Naples “Federico II”, 80131 Naples, Italy; mario.tortora@ymail.com (M.T.); mariotranfa@libero.it (M.T.); deliaannachiara@gmail.com (A.C.D.); giuseppe.pon@gmail.com (G.P.); brunetti@unina.it (A.B.); aelefant@unina.it (A.E.)

2 Department of Neurosciences and Reproductive and Odontostomatological Sciences, University “Federico II", 80131 Naples, Italy; maria.petracca@unina.it

3 Department of Human Neurosciences, Sapienza University of Rome, 00189 Rome, Italy

4 Neuroradiology Unit, NESMOS Department, Sapienza University of Rome, 00189 Rome, Italy; alessandro.bozzao@uniroma1.it

5 Department of Medicine of Precision, University of Campania “Luigi Vanvitelli”, 80138 Naples, Italy; ferdinando.caranci@unicampania.it

6 Department of Neuroradiology, ASST Grande Ospedale Metropolitano Niguarda, 20121 Milan, Italy; amedeo.cervo@gmail.com

7 Department of Translational Research and New Technologies in Medicine and Surgery, University of Pisa, 56126 Pisa, Italy; mirco.cosottini@unipi.it

check for updates

Citation: Tortora, M.; Tranfa, M.; D'Elia, A.C.; Pontillo, G.; Petracca, M.; Bozzao, A.; Caranci, F.; Cervo, A.; Cosottini, M.; Falini, A.; et al. Walk Your Talk: Real-World Adherence to Guidelines on the Use of MRI in Multiple Sclerosis. Diagnostics 2021, 11, 1310. https://doi.org/10.3390/ diagnostics 11081310

Academic Editor: Christoph Trumm

Received: 12 June 2021

Accepted: 19 July 2021

Published: 21 July 2021

Publisher's Note: MDPI stays neutral with regard to jurisdictional claims in published maps and institutional affiliations.

Copyright: (C) 2021 by the authors. Licensee MDPI, Basel, Switzerland. This article is an open access article distributed under the terms and conditions of the Creative Commons Attribution (CC BY) license (https:// creativecommons.org/licenses/by/ $4.0 /)$.
8 Neuroradiology Department, IRCCS San Raffaele Hospital and University, 20132 Milan, Italy; falini.andrea@unisr.it

9 Neuroradiology Unit, Department of Biomedical Sciences and Morphological and Functional Images, University of Messina, 98124 Messina, Italy; mlongo@unime.it

10 Department of Neurosciences, University of Padua, 35121 Padua, Italy; renzo.manara@unipd.it

11 Diagnostic and Interventional Neuroradiology, Cardarelli Hospital, 80131 Naples, Italy; mario.muto@aocardarelli.it

12 Department of Radiology, Azienda Ospedaliero Universitaria (A.O.U.) di Cagliari, 09124 Cagliari, Italy; micheleporcu87@gmail.com (M.P.); lucasabamd@gmail.com (L.S.)

13 Department of Health Sciences, University of Genova, 16132 Genova, Italy; lroccatagliata@neurologia.unige.it

14 Neuroradiology Unit, IRCCS Ospedale Policlinico San Martino, 16132 Genova, Italy

15 Neuroradiology Unit, Department of Neuroscience, Nuovo Ospedale Civile S. Agostino Estense, 41126 Modena, Italy; todeschini.ale@gmail.com

* Correspondence: sirio.cocozza@unina.it

+ These authors contributed equally to this work.

Abstract: (1) Although guidelines about the use of MRI sequences for Multiple Sclerosis (MS) diagnosis and follow-up are available, variability in acquisition protocols is not uncommon in everyday clinical practice. The aim of this study was to evaluate the real-world application of MS imaging guidelines in different settings to clarify the level of adherence to these guidelines. (2) Via an on-line anonymous survey, neuroradiologists (NR) were asked about MRI protocols and parameters routinely acquired when MS patients are evaluated in their center, both at diagnosis and followup. Furthermore, data about report content and personal opinions about emerging neuroimaging markers were also retrieved. (3) A total of 46 participants were included, mostly working in a hospital or university hospital (80.4\%) and with more than 10 years of experience $(47.9 \%)$. We found a relatively good adherence to the suggested MRI protocols regarding the use of T2-weighted sequences, although almost $10 \%$ of the participants routinely acquired $2 \mathrm{D}$ sequences with a slice thickness superior to $3 \mathrm{~mm}$. On the other hand, a wider degree of heterogeneity was found regarding gadolinium administration, almost routinely performed at follow-up examination (87.0\% of cases) in contrast with the current guidelines, as well as a low use of a standardized reporting system $(17.4 \%$ of cases). (4) Although the MS community is getting closer to a standardization of MRI protocols, there is still a relatively wide heterogeneity among NR, with particular reference to contrast administration, which must be overcome to guarantee an adequate quality of patients' care in MS. 
Keywords: MRI; multiple sclerosis; neuroradiology

\section{Introduction}

Multiple sclerosis (MS) is an autoimmune inflammatory disease affecting the central nervous system (CNS), leading to white matter (WM) demyelination, gray matter (GM) atrophy, and global neurodegeneration [1]. It shows a higher incidence in young adults, especially women, and it is characterized by a heterogeneous spectrum of symptoms and clinical phenotypes, which correspond to a variable degree of neurological disability [2]. Given this relatively wide heterogeneity in clinical presentation, there are many pathological entities that have to be considered and excluded in MS differential diagnosis [3]. The role of Magnetic Resonance Imaging (MRI) in supporting MS diagnosis has been clearly established over the past years. Indeed, the McDonald diagnostic criteria (from their first draft in 2001 to their latest revision in 2017) [4] are based on the demonstration of dissemination in space (DIS) and time (DIT) using MRI or through clinical symptoms and cerebrospinal fluid (CSF) markers, respectively. However, not only from a clinical but also from an MRI perspective, several conditions can mimic MS [5]. In this light, in order to facilitate the application of the McDonalds diagnostic criteria to avoiding misdiagnosis and to favor the MRI monitoring of disease activity over time [6], specific imaging protocols have been developed and are periodically revised and refined by experts in the field [7,8]. In Europe, a central role is played by the Magnetic Resonance Imaging in MS (MAGNIMS) guidelines, drafted by a network of MS experts [9]. Unfortunately, even though the MAGNIMS consensus guidelines clearly define the MRI sequences mandatory for both baseline and follow-up examinations [6,10], variability in MRI acquisition protocols is not an uncommon event in everyday clinical practice. In order to clarify the level of adherence to published guidelines [9] in clinical settings, we developed an anonymous online survey. Neuroradiologists (NR) working in different and relatively heterogeneous settings (ranging from university hospitals to private medical practice) were asked about the protocols applied for MS diagnosis/monitoring and about the study report produced for clinical purposes, which ideally should contain all the information necessary to allow diagnosis and monitor response to therapy.

\section{Materials and Methods}

For the dissemination of the questionnaire, based on our connections, we reached at least three neuroradiologists (one for each setting explored) per each Italian region in an attempt to enroll a representative sample. We then asked each reached NR who showed interest in participating in this work to also disseminate the survey to colleagues working in different centers, with the aim of increasing the sample size. The questionnaire was published online via the EUSurvey website (https: / / ec.europa.eu/ eusurvey accessed on 1 May 2021), and each participant was asked to respond according to their real-life experience rather than their theoretical knowledge of the current guidelines. The questionnaire, redacted in Italian, was fully anonymous and designed to be completed in 10 to $15 \mathrm{~min}$, presenting different sets of questions regarding the participant's professional profile, the MRI protocols used for diagnosis and follow up of MS, and the format and content of the study report. The complete questionnaire, translated from Italian to English, is available in Supplementary Materials. Briefly, the questionnaire was created to first obtain information about the participant's professional background (e.g., by retrieving information about the respondent's level of experience or where he/she usually performs the neuroradiological evaluation). A second set of questions covered the MRI protocol used for MS diagnosis, including the field strength used to evaluate these patients, the type of sequences used, and some additional information about their spatial resolution or time relation with gadolinium administration. The same questions were then asked regarding the neuroradiological practice at the follow-up examination. After the questions regarding the technical details of 
the acquisition, information about the neuroradiological report were retrieved. Finally, NRs were asked their opinion about some possible future directions of clinical neuroimaging in MS, such as the use of a new emerging sign or the application of more quantitative biomarkers of disease, such as the inclusion of an automatically generated report of the degree of brain atrophy. The Survey was published online for approximately 3 months from 9 February 2021. On 19 April 2021, the enrollment phase was considered closed. Survey responses were then retrieved end exported from the website as a.csv file, from which descriptive statistics were calculated. Results are presented for the entire group of participants, as well as stratified by years of experience and workplace (baseline acquisition only), in order to evaluate possible differences in terms of application depending on the work environment.

\section{Results}

\subsection{Participants}

Forty-six NRs completed the online survey, including 2 residents (4.3\%), 18 young NRs (39.1\%) (defined as board-certified NRs with less than 5 years of experience), 4 NRs $(8.7 \%)$ (defined as board-certified NRs with more than 5 years but less than 10 years of experience), and 22 NRs (47.9\%) with more than 10 years of experience. A large proportion of participants worked in a hospital or university hospital $(37 / 46,80.4 \%)$, with the remaining 9 NRs (19.6\%) evaluating MS patients in private clinical practice. More than $50 \%$ of the respondents $(52.2 \%)$ declared that they evaluate, on average, more than $10 \mathrm{MS}$ cases per month, in most cases $(67.4 \%)$ using a $1.5 \mathrm{~T}$ scanner. Information about participants' experience and work setting are displayed in Figure 1.

\section{Participants' information}
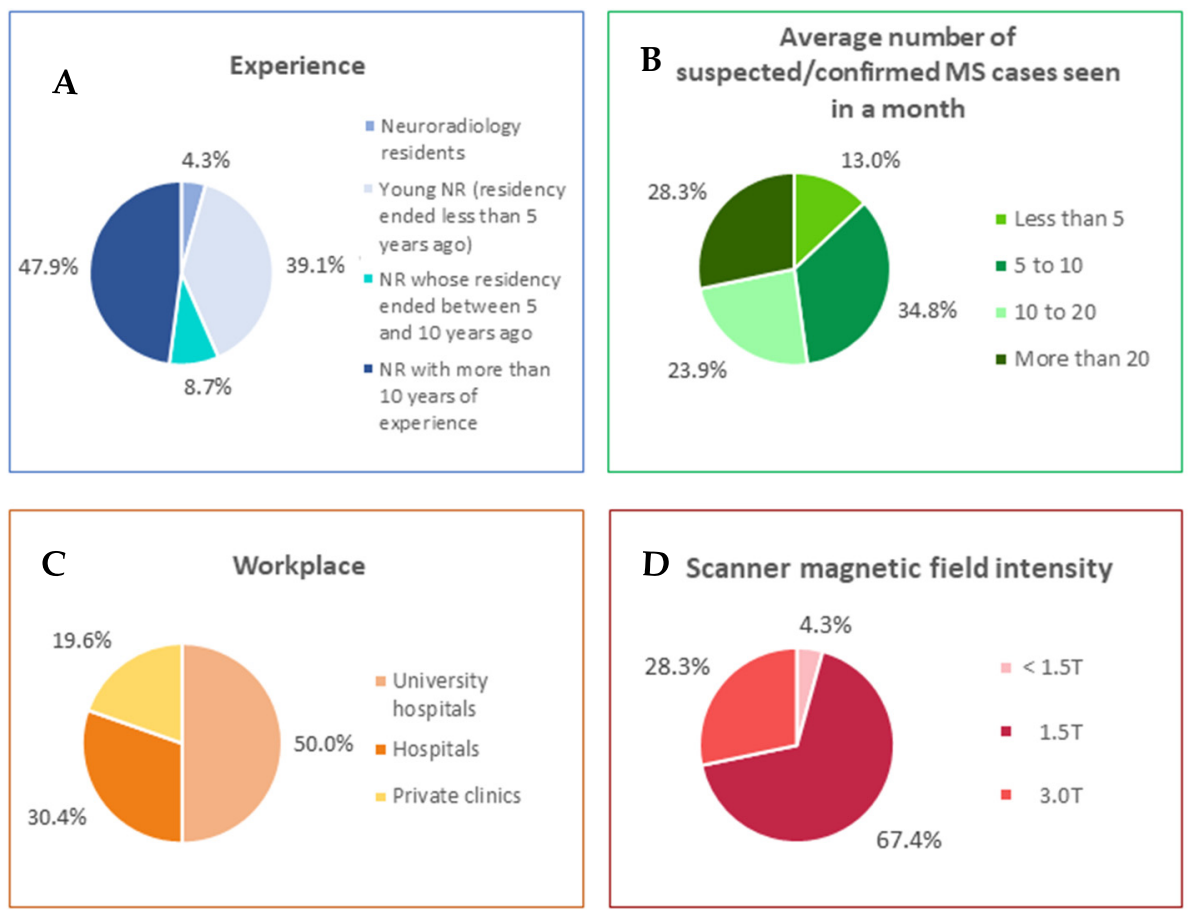

Figure 1. Participants' information. Pie charts represent (A) experience in neuroradiology field; (B) average number of suspected/confirmed Multiple Sclerosis cases usually seen in a month;

(C) workplace; (D) scanner magnetic field intensity. Data are show as percentages.

\subsection{Brain and Spine MRI Protocols-Baseline}

Regarding the baseline evaluation of MS patients, the majority of participants acquired a 3D fluid attenuated inversion recovery (FLAIR) volume $(40 / 46,87.0 \%)$, while the remaining opted for a 2D FLAIR sequence $(6 / 46,13.0 \%)$, with a slice thickness superior to $3 \mathrm{~mm}$ 
in almost all cases $(5 / 6,83.3 \%)$. Among the other T2-weighted sequences, the second-most acquired was the 2D T2w sequence (30/46, 65.2\%), with a slice thickness superior to $3 \mathrm{~mm}$ in half of the cases $(15 / 30,50.0 \%)$, while only few participants $(3 / 46,6.5 \%)$ acquired a $3 \mathrm{D} \mathrm{T} 2 \mathrm{w}$ volume. Among pre-contrast T1w sequences, 2D spin-echo (SE)-T1w was the most frequently reported ( $65.2 \%$ of the cases), followed by 3D gradient-echo (GrE)-T1w volumes (28.3\%). After gadolinium administration, the use of the two sequences was better balanced but still in favor of the 2D acquisition (2D SE-T1w images in $56.5 \%$ of the cases and 3D GrE-T1 $w$ volumes in 50.0\% of the cases). In the majority of cases $(28 / 41,68.3 \%)$, the post-gadolinium sequence was acquired $5 \mathrm{~min}$ after the end of contrast administration. A small percentage of participants $(5 / 46,10.9 \%)$ declared to not routinely acquire post-gadolinium sequences at baseline. At baseline, half of the participants routinely acquired a specific sequence for cortical lesions detection $(23 / 46,50.0 \%)$, with the $3 \mathrm{D}$ double inversion recovery (DIR) being the preferred choice $(14 / 23,60.9 \%)$. More than $80 \%$ of the respondents (38/46, 82.6\%) routinely acquired a sequence for optic nerves evaluation, with short tau inversion recovery (STIR)-T2w being the most common choice $(34 / 38,89.5 \%)$. In the framework of optic nerves evaluation, 20 participants also routinely acquired a post-gadolinium fat-saturated $\mathrm{T} 1 \mathrm{w}$ sequence $(58.8 \%)$. Finally, with reference to the spine MRI protocol at baseline, all participants acquired either a T2w or a STIR-T2w sagittal sequence, with a slice thickness inferior or equal to $3 \mathrm{~mm}$ in almost all cases $(44 / 46,95.6 \%)$. Sixty-three percent of the participants $(29 / 46)$ acquired a T1w sequence both before and after contrast administration, while $16 \mathrm{NRs}(34.8 \%)$ acquired a post-gadolinium sequence only. In almost all cases, spine T1w sequences had a 2D resolution $(43 / 46,93.5 \%)$. Of these, only $23(60.5 \%)$ had a thickness equal or inferior to $3 \mathrm{~mm}$ without a gap. Results relative to the baseline MRI protocol are summarized in Table 1 and Figure 2.

Table 1. Results of the survey regarding brain and spine MRI at baseline.

\begin{tabular}{|c|c|c|c|c|c|c|}
\hline \multicolumn{7}{|c|}{ BASELINE } \\
\hline \multicolumn{7}{|c|}{ BRAIN MRI } \\
\hline $\begin{array}{c}\text { MRI } \\
\text { SEQUENCES }\end{array}$ & $\begin{array}{c}\text { Number of Affirmative } \\
\text { Answers }\end{array}$ & Percentage & \multicolumn{2}{|c|}{ Additional Questions } & $\begin{array}{l}\text { Number of } \\
\text { Answers }\end{array}$ & Percentage \\
\hline \multicolumn{7}{|c|}{ T1-weighted } \\
\hline \multirow{4}{*}{$\begin{array}{l}\text { 2D SE-T1w } \\
\text { (pre-Gd) }\end{array}$} & \multirow{4}{*}{30} & \multirow{4}{*}{$65.2 \%$} & \multirow{2}{*}{ slice thickness } & $\leq 3 \mathrm{~mm}$ & 8 & $26.7 \%$ \\
\hline & & & & $>3 \mathrm{~mm}$ & 22 & $73.3 \%$ \\
\hline & & & \multirow{2}{*}{ gap } & Yes & 10 & $33.3 \%$ \\
\hline & & & & No & 20 & $66.7 \%$ \\
\hline \multirow{3}{*}{$\begin{array}{l}\text { 3D SE-T1w } \\
\text { (pre-Gd) }\end{array}$} & \multirow{3}{*}{6} & \multirow{3}{*}{$13.0 \%$} & \multirow{3}{*}{ voxel size } & $<1 \mathrm{~mm}$ (isotropic) & 3 & $50.0 \%$ \\
\hline & & & & $1 \mathrm{~mm}$ (isotropic) & 3 & $50.0 \%$ \\
\hline & & & & other & 0 & $0.0 \%$ \\
\hline \multirow{3}{*}{$\begin{array}{l}\text { 3D GrE-T1w } \\
\text { (pre-Gd) }\end{array}$} & \multirow{3}{*}{13} & \multirow{3}{*}{$28.3 \%$} & \multirow{3}{*}{ voxel size } & $<1 \mathrm{~mm}$ (isotropic) & 1 & $7.7 \%$ \\
\hline & & & & $1 \mathrm{~mm}$ (isotropic) & 12 & $92.3 \%$ \\
\hline & & & & other & 0 & $0.0 \%$ \\
\hline \multirow{6}{*}{$\begin{array}{l}\text { 2D SE-T1w } \\
\text { (post-Gd) }\end{array}$} & \multirow{6}{*}{26} & \multirow{6}{*}{$56.5 \%$} & \multirow{2}{*}{ slice thickness } & $\leq 3 \mathrm{~mm}$ & 10 & $38.5 \%$ \\
\hline & & & & $>3 \mathrm{~mm}$ & 16 & $61.5 \%$ \\
\hline & & & \multirow{2}{*}{ gap } & Yes & 11 & $42.3 \%$ \\
\hline & & & & No & 15 & $57.7 \%$ \\
\hline & & & \multirow{2}{*}{ delay } & $\leq 5^{\prime}$ & 11 & $42.3 \%$ \\
\hline & & & & $>5^{\prime}$ & 15 & $57.7 \%$ \\
\hline
\end{tabular}


Table 1. Cont.

\begin{tabular}{|c|c|c|c|c|c|c|}
\hline \multirow[t]{2}{*}{$\begin{array}{l}\text { 3D SE-T1w } \\
\text { (post-Gd) }\end{array}$} & \multirow[t]{2}{*}{16} & \multirow[t]{2}{*}{$34.8 \%$} & voxel size & $\begin{array}{c}<1 \mathrm{~mm} \text { (isotropic) } \\
1 \mathrm{~mm} \text { (isotropic) } \\
\text { other }\end{array}$ & $\begin{array}{l}6 \\
8 \\
2 \\
\end{array}$ & $\begin{array}{l}37.5 \% \\
50.0 \% \\
12.5 \% \\
\end{array}$ \\
\hline & & & delay & $\begin{array}{l}\leq 5^{\prime} \\
>5^{\prime}\end{array}$ & $\begin{array}{c}11 \\
5\end{array}$ & $\begin{array}{l}68.7 \% \\
31.3 \% \\
\end{array}$ \\
\hline \multirow[t]{2}{*}{$\begin{array}{l}\text { 3D GrE-T1w } \\
\text { (post-Gd) }\end{array}$} & \multirow[t]{2}{*}{23} & \multirow[t]{2}{*}{$50.0 \%$} & voxel size & $\begin{array}{c}<1 \mathrm{~mm} \text { (isotropic) } \\
1 \mathrm{~mm} \text { (isotropic) } \\
\text { other }\end{array}$ & $\begin{array}{c}3 \\
18 \\
1 \\
\end{array}$ & $\begin{array}{c}13.6 \% \\
81.8 \% \\
4.6 \% \\
\end{array}$ \\
\hline & & & delay & $\begin{array}{l}\leq 5^{\prime} \\
>5^{\prime}\end{array}$ & $\begin{array}{c}7 \\
15\end{array}$ & $\begin{array}{l}31.8 \% \\
68.2 \%\end{array}$ \\
\hline \multirow{3}{*}{$\mathrm{PD} / \mathrm{T} 2 \mathrm{w}$} & & & T2-weighted & & & \\
\hline & \multirow{2}{*}{1} & \multirow{2}{*}{$2.2 \%$} & slice thickness & $\begin{array}{l}\leq 3 \mathrm{~mm} \\
>3 \mathrm{~mm}\end{array}$ & $\begin{array}{l}1 \\
0\end{array}$ & $\begin{array}{l}100 \% \\
0.0 \%\end{array}$ \\
\hline & & & gap & $\begin{array}{l}\text { Yes } \\
\text { No }\end{array}$ & $\begin{array}{l}0 \\
1\end{array}$ & $\begin{array}{c}0.0 \% \\
100.0 \%\end{array}$ \\
\hline \multirow{2}{*}{ 2D T2w } & \multirow{2}{*}{30} & \multirow{2}{*}{$65.2 \%$} & slice thickness & $\begin{array}{l}\leq 3 \mathrm{~mm} \\
>3 \mathrm{~mm}\end{array}$ & $\begin{array}{l}15 \\
15\end{array}$ & $\begin{array}{l}50.0 \% \\
50.0 \% \\
\end{array}$ \\
\hline & & & gap & $\begin{array}{l}\text { Yes } \\
\text { No }\end{array}$ & $\begin{array}{l}13 \\
17\end{array}$ & $\begin{array}{l}43.3 \% \\
56.7 \%\end{array}$ \\
\hline 3D T2w & 3 & $6.5 \%$ & voxel size & $\begin{array}{c}<1 \mathrm{~mm} \text { (isotropic) } \\
1 \mathrm{~mm} \text { (isotropic) } \\
\text { other }\end{array}$ & $\begin{array}{l}1 \\
1 \\
1\end{array}$ & $\begin{array}{l}33.3 \% \\
33.3 \% \\
33.3 \%\end{array}$ \\
\hline \multirow{2}{*}{ 2D FLAIR } & \multirow{2}{*}{12} & \multirow{2}{*}{$26.1 \%$} & slice thickness & $\begin{array}{l}\leq 3 \mathrm{~mm} \\
>3 \mathrm{~mm}\end{array}$ & $\begin{array}{l}4 \\
8 \\
\end{array}$ & $\begin{array}{l}33.3 \% \\
66.7 \% \\
\end{array}$ \\
\hline & & & gap & $\begin{array}{l}\text { Yes } \\
\text { No }\end{array}$ & $\begin{array}{c}2 \\
10\end{array}$ & $\begin{array}{l}16.7 \% \\
83.3 \%\end{array}$ \\
\hline 3D FLAIR & 40 & $87.0 \%$ & voxel size & $\begin{array}{c}<1 \mathrm{~mm} \text { (isotropic) } \\
1 \mathrm{~mm} \text { (isotropic) } \\
\text { other }\end{array}$ & $\begin{array}{c}10 \\
24 \\
6 \\
\end{array}$ & $\begin{array}{l}25.0 \% \\
60.0 \% \\
15.0 \% \\
\end{array}$ \\
\hline \multicolumn{7}{|c|}{ Sequences for cortical lesions detection } \\
\hline \multirow{2}{*}{ 2D DIR } & \multirow{2}{*}{3} & \multirow{2}{*}{$6.5 \%$} & slice thickness & $\begin{array}{l}\leq 3 \mathrm{~mm} \\
>3 \mathrm{~mm}\end{array}$ & $\begin{array}{l}2 \\
1\end{array}$ & $\begin{array}{l}66.7 \% \\
33.3 \%\end{array}$ \\
\hline & & & gap & $\begin{array}{l}\text { Yes } \\
\text { No }\end{array}$ & $\begin{array}{l}1 \\
2\end{array}$ & $\begin{array}{l}33.3 \% \\
66.7 \%\end{array}$ \\
\hline 3D DIR & 14 & $30.4 \%$ & voxel size & $\begin{array}{c}<1 \mathrm{~mm} \text { (isotropic) } \\
1 \mathrm{~mm} \text { (isotropic) } \\
\text { other }\end{array}$ & $\begin{array}{c}2 \\
10 \\
2\end{array}$ & $\begin{array}{l}14.3 \% \\
71.4 \% \\
14.3 \%\end{array}$ \\
\hline 2D PSIR & 4 & $8.7 \%$ & slice thickness & $\begin{array}{l}\leq 3 \mathrm{~mm} \\
>3 \mathrm{~mm}\end{array}$ & $\begin{array}{l}2 \\
2 \\
\end{array}$ & $\begin{array}{l}50.0 \% \\
50.0 \% \\
\end{array}$ \\
\hline & & & gap & $\begin{array}{l}\text { Yes } \\
\text { No }\end{array}$ & $\begin{array}{l}2 \\
2\end{array}$ & $\begin{array}{l}50.0 \% \\
50.0 \%\end{array}$ \\
\hline 3D PSIR & 3 & $6.5 \%$ & voxel size & $\begin{array}{c}<1 \mathrm{~mm} \text { (isotropic) } \\
1 \mathrm{~mm} \text { (isotropic) } \\
\text { other }\end{array}$ & $\begin{array}{l}0 \\
3 \\
0\end{array}$ & $\begin{array}{c}0.0 \% \\
100 \% \\
0.0 \%\end{array}$ \\
\hline None & 23 & $50.0 \%$ & & & & \\
\hline
\end{tabular}


Table 1. Cont.

\begin{tabular}{|c|c|c|c|c|c|c|}
\hline \multicolumn{7}{|c|}{ Sequences for optic nerves evaluation } \\
\hline \multirow{4}{*}{ STIR-T2w } & \multirow{4}{*}{34} & \multirow{4}{*}{$73.9 \%$} & \multirow{2}{*}{ slice thickness } & $\leq 3 \mathrm{~mm}$ & 31 & $91.2 \%$ \\
\hline & & & & $>3 \mathrm{~mm}$ & 3 & $8.8 \%$ \\
\hline & & & \multirow{2}{*}{ gap } & Yes & 10 & $29.4 \%$ \\
\hline & & & & No & 24 & $70.6 \%$ \\
\hline \multirow{4}{*}{$\begin{array}{c}\text { Post-Gd Fat-Sat } \\
\text { T1w }\end{array}$} & \multirow{4}{*}{22} & \multirow{4}{*}{$47.8 \%$} & \multirow{2}{*}{ slice thickness } & $\leq 3 \mathrm{~mm}$ & 22 & $100 \%$ \\
\hline & & & & $>3 \mathrm{~mm}$ & 0 & $0.0 \%$ \\
\hline & & & \multirow{2}{*}{ gap } & Yes & 5 & $22.7 \%$ \\
\hline & & & & No & 17 & $77.3 \%$ \\
\hline None & 8 & $17.4 \%$ & & & & \\
\hline \multicolumn{7}{|c|}{ Additional sequences } \\
\hline DWI & 44 & $95.6 \%$ & & & & \\
\hline \multirow{4}{*}{ GrE-T2* } & \multirow{4}{*}{17} & \multirow{4}{*}{$37.0 \%$} & \multirow{2}{*}{ slice thickness } & $\leq 3 \mathrm{~mm}$ & 4 & $23.5 \%$ \\
\hline & & & & $>3 \mathrm{~mm}$ & 13 & $76.5 \%$ \\
\hline & & & \multirow{2}{*}{ gap } & Yes & 8 & $47.1 \%$ \\
\hline & & & & No & 9 & $53.9 \%$ \\
\hline \multirow{4}{*}{ 2D SWI } & \multirow{4}{*}{12} & \multirow{4}{*}{$26.1 \%$} & \multirow{2}{*}{ slice thickness } & $\leq 3 \mathrm{~mm}$ & 9 & $75.0 \%$ \\
\hline & & & & $>3 \mathrm{~mm}$ & 3 & $25.0 \%$ \\
\hline & & & \multirow{2}{*}{ gap } & Yes & 2 & $16.7 \%$ \\
\hline & & & & No & 10 & $83.3 \%$ \\
\hline \multirow{3}{*}{ 3D SWI } & & & & $<1 \mathrm{~mm}$ (isotropic) & 4 & $26.7 \%$ \\
\hline & 15 & $32.6 \%$ & voxel size & $1 \mathrm{~mm}$ (isotropic) & 9 & $60.0 \%$ \\
\hline & & & & other & 2 & $13.3 \%$ \\
\hline None & 1 & $2.2 \%$ & & & & \\
\hline & & & PINE MRI & & & \\
\hline $\begin{array}{c}\text { MRI } \\
\text { SEQUENCES }\end{array}$ & $\begin{array}{c}\text { Number of Affirmative } \\
\text { Answers }\end{array}$ & Percentage & Additional & Destions & $\begin{array}{l}\text { Number of } \\
\text { Answers }\end{array}$ & Percentage \\
\hline & & & 2-weighted & & & \\
\hline & & & acquisition plane & sagittal & 41 & $100 \%$ \\
\hline & & & acquistiont prane & axial & 17 & $41.5 \%$ \\
\hline & & & slice thickness on & $\leq 3 \mathrm{~mm}$ & 39 & $95.1 \%$ \\
\hline $12 \mathrm{w}$ & 41 & $89.1 \%$ & sagittal acquisition & $>3 \mathrm{~mm}$ & 2 & $4.9 \%$ \\
\hline & & & gap on sagittal & Yes & 14 & $34.1 \%$ \\
\hline & & & acquisition & No & 27 & $65.9 \%$ \\
\hline & & & acquisition plane & sagittal & 2 & $100.0 \%$ \\
\hline & & & & axial & 0 & $0.0 \%$ \\
\hline & & & slice thickness on & $\leq 3 \mathrm{~mm}$ & 1 & $50.0 \%$ \\
\hline PDW & 2 & $4.3 \%$ & sagittal acquisition & $>3 \mathrm{~mm}$ & 1 & $50.0 \%$ \\
\hline & & & gap on sagittal & Yes & 2 & $100.0 \%$ \\
\hline & & & acquisition & No & 0 & $0.0 \%$ \\
\hline & & & acquisition plane & sagittal & 42 & $100.0 \%$ \\
\hline & & & acquistiont piante & axial & 2 & $4.7 \%$ \\
\hline & & & slice thickness on & $\leq 3 \mathrm{~mm}$ & 38 & $90.5 \%$ \\
\hline$S H 1 / 2-12 \mathrm{w}$ & 42 & $91.3 \%$ & sagittal acquisition & $>3 \mathrm{~mm}$ & 4 & $9.5 \%$ \\
\hline & & & gap on sagittal & Yes & 14 & $33.3 \%$ \\
\hline & & & acquisition & No & 28 & $66.7 \%$ \\
\hline
\end{tabular}


Table 1. Cont.

\begin{tabular}{|c|c|c|c|c|c|c|}
\hline \multirow{7}{*}{ PSIR } & & T1-weig & and inversion recove & & & \\
\hline & \multirow{6}{*}{2} & \multirow{6}{*}{$4.3 \%$} & \multirow{2}{*}{ acquisition plane } & sagittal & 2 & $100 \%$ \\
\hline & & & & axial & 0 & $0.0 \%$ \\
\hline & & & \multirow{2}{*}{$\begin{array}{l}\text { slice thickness on } \\
\text { sagittal acquisition }\end{array}$} & $\leq 3 \mathrm{~mm}$ & 2 & $100 \%$ \\
\hline & & & & $>3 \mathrm{~mm}$ & 0 & $0.0 \%$ \\
\hline & & & \multirow{2}{*}{$\begin{array}{l}\text { gap on sagittal } \\
\text { acquisition }\end{array}$} & Yes & 1 & $50.0 \%$ \\
\hline & & & & No & 1 & $50.0 \%$ \\
\hline \multirow{6}{*}{$\begin{array}{l}\text { 2D SE-T1w } \\
\text { (pre-Gd) }\end{array}$} & \multirow{6}{*}{26} & \multirow{6}{*}{$56.5 \%$} & \multirow{2}{*}{ acquisition plane } & sagittal & 26 & $100 \%$ \\
\hline & & & & axial & 3 & $11.5 \%$ \\
\hline & & & \multirow{2}{*}{$\begin{array}{l}\text { slice thickness on } \\
\text { sagittal acquisition }\end{array}$} & $\leq 3 \mathrm{~mm}$ & 23 & $88.5 \%$ \\
\hline & & & & $>3 \mathrm{~mm}$ & 3 & $11.5 \%$ \\
\hline & & & \multirow{2}{*}{$\begin{array}{l}\text { gap on sagittal } \\
\text { acquisition }\end{array}$} & Yes & 11 & $42.3 \%$ \\
\hline & & & & No & 15 & $57.7 \%$ \\
\hline \multirow{6}{*}{$\begin{array}{l}\text { 3D GrE-T1w } \\
\text { (pre-Gd) }\end{array}$} & \multirow{6}{*}{2} & \multirow{6}{*}{$4.3 \%$} & \multirow{2}{*}{ acquisition plane } & sagittal & 2 & $100 \%$ \\
\hline & & & & axial & 1 & $50.0 \%$ \\
\hline & & & \multirow{2}{*}{$\begin{array}{l}\text { slice thickness on } \\
\text { sagittal acquisition }\end{array}$} & $\leq 3 \mathrm{~mm}$ & 2 & $100 \%$ \\
\hline & & & & $>3 \mathrm{~mm}$ & 0 & $0.0 \%$ \\
\hline & & & \multirow{2}{*}{$\begin{array}{l}\text { gap on sagittal } \\
\text { acquisition }\end{array}$} & Yes & 0 & $0.0 \%$ \\
\hline & & & & No & 2 & $100 \%$ \\
\hline \multirow{8}{*}{$\begin{array}{l}\text { 2D SE-T1w } \\
\text { (post-Gd) }\end{array}$} & \multirow{8}{*}{42} & \multirow{8}{*}{$91.3 \%$} & \multirow{2}{*}{ acquisition plane } & sagittal & 41 & $100 \%$ \\
\hline & & & & axial & 13 & $31.7 \%$ \\
\hline & & & \multirow{2}{*}{$\begin{array}{l}\text { slice thickness on } \\
\text { sagittal acquisition }\end{array}$} & $\leq 3 \mathrm{~mm}$ & 37 & $90.2 \%$ \\
\hline & & & & $>3 \mathrm{~mm}$ & 4 & $9.8 \%$ \\
\hline & & & \multirow{2}{*}{$\begin{array}{l}\text { gap on sagittal } \\
\text { acquisition }\end{array}$} & Yes & 16 & $39.0 \%$ \\
\hline & & & & No & 25 & $61.0 \%$ \\
\hline & & & \multirow{2}{*}{ delay } & $<5^{\prime}$ & 19 & $47.5 \%$ \\
\hline & & & & $>5^{\prime}$ & 21 & $52.5 \%$ \\
\hline \multirow{8}{*}{$\begin{array}{l}\text { 3D GrE-T1w } \\
\text { (post-Gd) }\end{array}$} & \multirow{8}{*}{3} & & \multirow{2}{*}{ acquisition plane } & sagittal & 2 & $66.7 \%$ \\
\hline & & & & axial & 2 & $66.7 \%$ \\
\hline & & & slice thickness on & $\leq 3 \mathrm{~mm}$ & 2 & $100 \%$ \\
\hline & & $65 \%$ & sagittal acquisition & $>3 \mathrm{~mm}$ & 0 & $0.0 \%$ \\
\hline & & & gap on sagittal & Yes & 0 & $0.0 \%$ \\
\hline & & & acquisition & No & 2 & $100 \%$ \\
\hline & & & dolou & $\leq 5^{\prime}$ & 1 & $33.3 \%$ \\
\hline & & & delay & $>5^{\prime}$ & 2 & $66.7 \%$ \\
\hline
\end{tabular}

$\mathrm{SE}$ = spin-echo; GrE = gradient-echo; FLAIR = fluid attenuated inversion recovery; DIR = double inversion recovery; PSIR = phase sensitive inversion recovery; Fat-Sat $=$ fat saturated DWI = diffusion weighted imaging; $\mathrm{SWI}=$ susceptibility weighted imaging; $\mathrm{PD}=$ proton density; STIR = short tau inversion recovery; $\mathrm{Gd}=$ gadolinium . 


\section{BASELINE}

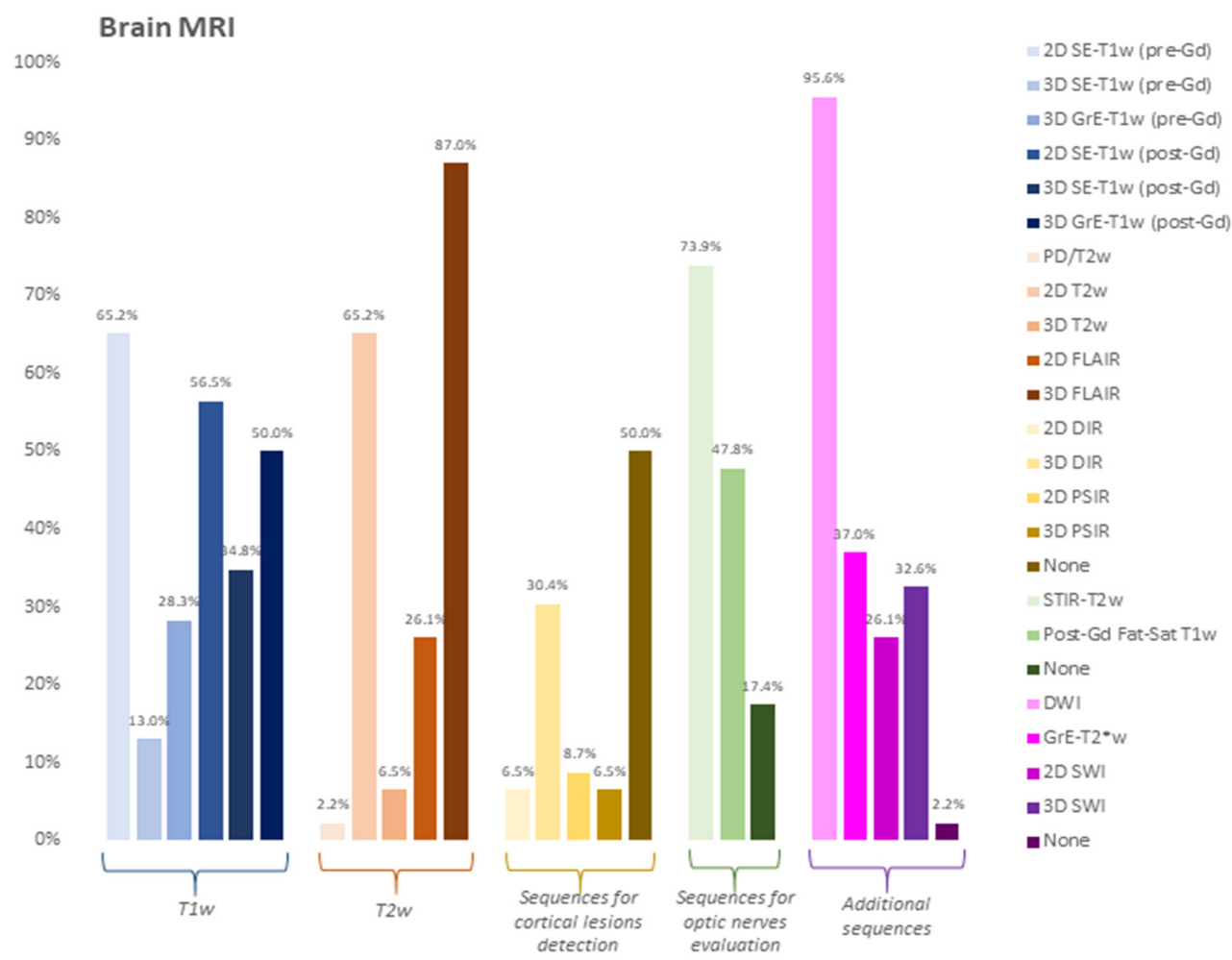

Spine MRI
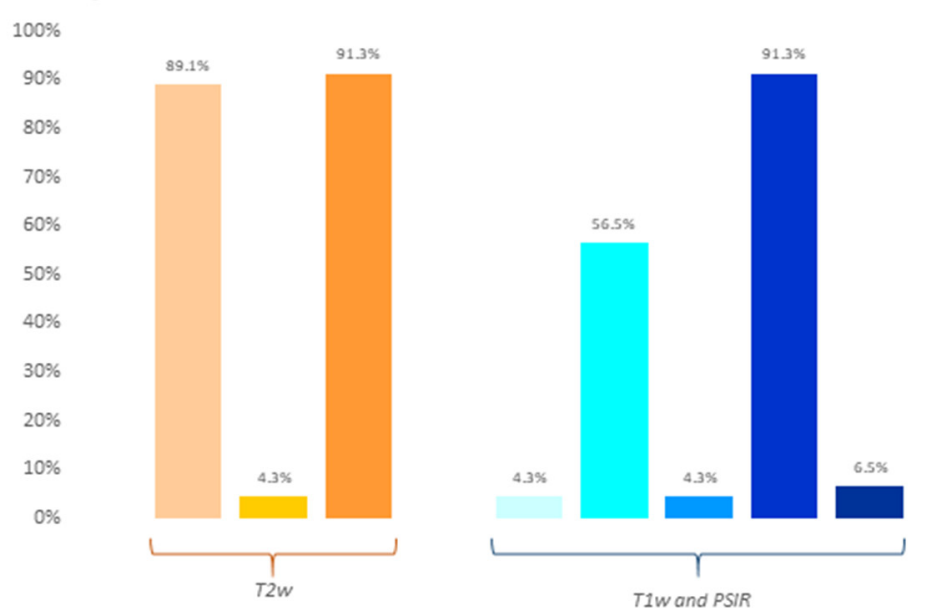

$m \mathrm{~T} 2 \mathrm{w}$

$=\mathrm{PDw}$

$=$ STIR-T2W

PSIR

$=2 \mathrm{DSE}-\mathrm{T} 1 \mathrm{w}$ (pre-Gd)

- 3D GrE-T1w (pre-Gd)

- 2D SE-T1w (post-Gd)

- 3D GrE-T1w (post-Gd)

Figure 2. Sequences included in the baseline brain and spine MRI protocols. Data are show as percentages. SE = spin-echo; GrE = gradient-echo; FLAIR = fluid attenuated inversion recovery; DIR = double inversion recovery; PSIR = phase sensitive inversion recovery; Fat-Sat = fat saturated; DWI = diffusion weighted imaging; SWI = susceptibility weighted imaging; $\mathrm{PD}=$ proton density; STIR = short tau inversion recovery; $\mathrm{Gd}=$ gadolinium .

\subsection{Brain and Spine MRI Protocols—Follow-Up}

Regarding the brain acquisition at a follow-up examination, it was confirmed that most of the participants acquired a 3D FLAIR sequence (37/46, 80.4\%), in line with data obtained from baseline evaluation. Similarly, among the other T2w images as well, the second-most used was the 2D T2w sequence $(31 / 46,67.4 \%)$, with a thickness superior to $3 \mathrm{~mm}$ in about half cases $(15 / 31,48.4 \%)$. Finally, the use of pre-contrast T1w sequences was also in line with the baseline (SE-T1w: 28/46, 60.9\%; 3D GrE-T1w: 10/46, 21.7\%). 
The vast majority of participants $(40 / 46,87.0 \%)$ declared that they perform at least one post-contrast T1w sequence, either a 3D GrE-T1w (23/40, 57.5\%) or a 2D SE-T1w sequence $(21 / 40,52.5 \%)$. Compared to the baseline evaluation, the acquisition of specific sequences for cortical lesions detection was slightly less frequent on follow-up examination (20/46 of cases, $43.5 \%)$, with the 3D DIR sequence remaining the NRs' preferred choice $(11 / 20$, $55.0 \%$ ). A reduction in the percentage of participants acquiring a specific sequence for optic nerve evaluation was also observed, with $24 / 46$ NR (52.2\%) acquiring a STIR-T2w sequence, followed in less than half cases by contrast administration $(11 / 24,45.8 \%)$. Finally, sequences acquired for spine evaluation at follow-up mirrored the one applied for the baseline evaluation in terms of T2w acquisition (43/46, 93.5\%). Interestingly, a similar stable pattern was observed in terms of contrast administration at follow-up, with 40/46 NRs $(87.0 \%)$ routinely administering Gadolinium for the execution of a post-contrast T1w sequence alone $(19 / 40,47.5 \%)$ or along with a pre-contrast acquisition $(21 / 40,52.5 \%)$. Results relative to the follow-up MRI protocol are summarized in Table 2 and Figure 3.

Table 2. Results of the survey regarding brain and spine MRI at follow-up.

\begin{tabular}{|c|c|c|c|c|c|c|}
\hline \multicolumn{7}{|c|}{ FOLLOW-UP } \\
\hline \multicolumn{7}{|c|}{ BRAIN MRI } \\
\hline $\begin{array}{c}\text { MRI } \\
\text { SEQUENCES }\end{array}$ & $\begin{array}{c}\text { Number of Affirmative } \\
\text { Answers }\end{array}$ & Percentage & \multicolumn{2}{|c|}{ Additional Questions } & $\begin{array}{l}\text { Number of } \\
\text { Answers }\end{array}$ & Percentage \\
\hline \multicolumn{7}{|c|}{ T1-weighted } \\
\hline \multirow{4}{*}{$\begin{array}{l}\text { 2D SE-T1w } \\
\text { (pre-Gd) }\end{array}$} & \multirow{4}{*}{28} & \multirow{4}{*}{$60.9 \%$} & \multirow{2}{*}{ slice thickness } & $\leq 3 \mathrm{~mm}$ & 10 & $35.7 \%$ \\
\hline & & & & $>3 \mathrm{~mm}$ & 18 & $64.3 \%$ \\
\hline & & & \multirow{2}{*}{ gap } & Yes & 12 & $42.9 \%$ \\
\hline & & & & No & 16 & $57.1 \%$ \\
\hline \multirow{3}{*}{$\begin{array}{l}\text { 3D SE-T1w } \\
\text { (pre-Gd) }\end{array}$} & \multirow{3}{*}{4} & \multirow{3}{*}{$8.7 \%$} & \multirow{3}{*}{ voxel size } & $<1 \mathrm{~mm}$ (isotropic) & 2 & $66.7 \%$ \\
\hline & & & & $1 \mathrm{~mm}$ (isotropic) & 1 & $33.3 \%$ \\
\hline & & & & other & 0 & $0.0 \%$ \\
\hline \multirow{3}{*}{$\begin{array}{l}\text { 3D GrE-T1w } \\
\text { (pre-Gd) }\end{array}$} & \multirow{3}{*}{10} & \multirow{3}{*}{$21.7 \%$} & \multirow{3}{*}{ voxel size } & $<1 \mathrm{~mm}$ (isotropic) & 1 & $10.0 \%$ \\
\hline & & & & $1 \mathrm{~mm}$ (isotropic) & 9 & $90.0 \%$ \\
\hline & & & & other & 0 & $0.0 \%$ \\
\hline \multirow{6}{*}{$\begin{array}{l}\text { 2D SE-T1w } \\
\text { (post-Gd) }\end{array}$} & \multirow{6}{*}{21} & \multirow{6}{*}{$45.6 \%$} & \multirow{2}{*}{ slice thickness } & $\leq 3 \mathrm{~mm}$ & 9 & $42.9 \%$ \\
\hline & & & & $>3 \mathrm{~mm}$ & 12 & $57.1 \%$ \\
\hline & & & \multirow{2}{*}{ gap } & Yes & 5 & $23.8 \%$ \\
\hline & & & & No & 16 & $76.2 \%$ \\
\hline & & & \multirow{2}{*}{ delay } & $\leq 5^{\prime}$ & 12 & $57.1 \%$ \\
\hline & & & & $>5^{\prime}$ & 8 & $38.1 \%$ \\
\hline \multirow{5}{*}{$\begin{array}{l}\text { 3D SE-T1w } \\
\text { (post-Gd) }\end{array}$} & \multirow{5}{*}{9} & \multirow{5}{*}{$19.6 \%$} & \multirow{3}{*}{ voxel size } & $<1 \mathrm{~mm}$ (isotropic) & 3 & $33.3 \%$ \\
\hline & & & & $1 \mathrm{~mm}$ (isotropic) & 5 & $55.5 \%$ \\
\hline & & & & other & 1 & $11.1 \%$ \\
\hline & & & \multirow{2}{*}{ delay } & $\leq 5^{\prime}$ & 6 & $66.6 \%$ \\
\hline & & & & $>5^{\prime}$ & 3 & $33.3 \%$ \\
\hline \multirow{5}{*}{$\begin{array}{l}\text { 3D GrE-T1w } \\
\text { (post-Gd) }\end{array}$} & \multirow{5}{*}{23} & \multirow{5}{*}{$50.0 \%$} & & $<1 \mathrm{~mm}$ (isotropic) & 3 & $13.0 \%$ \\
\hline & & & voxel size & $1 \mathrm{~mm}$ (isotropic) & 18 & $78.3 \%$ \\
\hline & & & & other & 0 & $0.0 \%$ \\
\hline & & & delay & $\leq 5^{\prime}$ & 5 & $21.7 \%$ \\
\hline & & & delay & $>5^{\prime}$ & 16 & $70.0 \%$ \\
\hline
\end{tabular}


Table 2. Cont

\begin{tabular}{|c|c|c|c|c|c|c|}
\hline \multicolumn{7}{|c|}{ T2-weighted } \\
\hline \multirow{4}{*}{$\mathrm{PD} / \mathrm{T} 2 \mathrm{w}$} & \multirow{4}{*}{1} & \multirow{4}{*}{$2.2 \%$} & \multirow{2}{*}{ slice thickness } & $\leq 3 \mathrm{~mm}$ & 0 & $0.0 \%$ \\
\hline & & & & $>3 \mathrm{~mm}$ & 1 & $100 \%$ \\
\hline & & & \multirow{2}{*}{ gap } & Yes & 0 & $0.0 \%$ \\
\hline & & & & No & 1 & $100 \%$ \\
\hline \multirow{4}{*}{ 2D T2w } & \multirow{4}{*}{31} & \multirow{4}{*}{$67.4 \%$} & \multirow{2}{*}{ slice thickness } & $\leq 3 \mathrm{~mm}$ & 14 & $45.2 \%$ \\
\hline & & & & $>3 \mathrm{~mm}$ & 15 & $48.4 \%$ \\
\hline & & & \multirow{2}{*}{ gap } & Yes & 11 & $35.5 \%$ \\
\hline & & & & No & 18 & $58.1 \%$ \\
\hline \multirow{3}{*}{$3 \mathrm{D} \mathrm{T} 2 \mathrm{w}$} & \multirow{3}{*}{1} & \multirow{3}{*}{$2.2 \%$} & \multirow{3}{*}{ voxel size } & $<1 \mathrm{~mm}$ (isotropic) & 1 & $100 \%$ \\
\hline & & & & $1 \mathrm{~mm}$ (isotropic) & 0 & $0.0 \%$ \\
\hline & & & & other & 0 & $0.0 \%$ \\
\hline \multirow{4}{*}{ 2D FLAIR } & \multirow{4}{*}{11} & \multirow{4}{*}{$23.9 \%$} & \multirow{2}{*}{ slice thickness } & $\leq 3 \mathrm{~mm}$ & 3 & $27.3 \%$ \\
\hline & & & & $>3 \mathrm{~mm}$ & 8 & $72.7 \%$ \\
\hline & & & \multirow{2}{*}{ gap } & Yes & 2 & $18.2 \%$ \\
\hline & & & & No & 9 & $81.8 \%$ \\
\hline \multirow{3}{*}{ 3D FLAIR } & \multirow{3}{*}{37} & \multirow{3}{*}{$80.4 \%$} & & $<1 \mathrm{~mm}$ (isotropic) & 9 & $24.3 \%$ \\
\hline & & & voxel size & $1 \mathrm{~mm}$ (isotropic) & 23 & $62.2 \%$ \\
\hline & & & & other & 5 & $13.5 \%$ \\
\hline & & quences & cortical lesions & ection & & \\
\hline & & & & $\leq 3 \mathrm{~mm}$ & 3 & $100 \%$ \\
\hline 2D DIR & 3 & $6.5 \%$ & slice thickness & $>3 \mathrm{~mm}$ & 0 & $0.0 \%$ \\
\hline & & & gap & Yes & 0 & $0.0 \%$ \\
\hline & & & gap & No & 3 & $100 \%$ \\
\hline & & & & $<1 \mathrm{~mm}$ (isotropic) & 3 & $27.3 \%$ \\
\hline 3D DIR & 11 & $23.9 \%$ & voxel size & $1 \mathrm{~mm}$ (isotropic) & 7 & $63.6 \%$ \\
\hline & & & & other & 1 & $9.1 \%$ \\
\hline & & & & $\leq 3 \mathrm{~mm}$ & 2 & $50.0 \%$ \\
\hline 2D PSIR & 4 & $87 \%$ & slice thickness & $>3 \mathrm{~mm}$ & 1 & $25.0 \%$ \\
\hline & & & gap & Yes & 2 & $50.0 \%$ \\
\hline & & & Sup & No & 1 & $25.0 \%$ \\
\hline & & & & $<1 \mathrm{~mm}$ (isotropic) & 1 & $33.3 \%$ \\
\hline 3D PSIR & 3 & $6.5 \%$ & voxel size & $1 \mathrm{~mm}$ (isotropic) & 2 & $66.7 \%$ \\
\hline & & & & other & 0 & $0.0 \%$ \\
\hline None & 27 & $58.7 \%$ & & & & \\
\hline & & equences & optic nerves ev & ation & & \\
\hline & & & slice thickness & $\leq 3 \mathrm{~mm}$ & 22 & $91.7 \%$ \\
\hline STIR-T2w & 24 & $522 \%$ & snce tnickness & $>3 \mathrm{~mm}$ & 2 & $8.3 \%$ \\
\hline & & & gap & Yes & 6 & $25.0 \%$ \\
\hline & & & gap & No & 18 & $75.0 \%$ \\
\hline & & & slice thickness & $\leq 3 \mathrm{~mm}$ & 16 & $100 \%$ \\
\hline Post-Gd Fat-Sat & 16 & $348 \%$ & snce tnickness & $>3 \mathrm{~mm}$ & 0 & $0.0 \%$ \\
\hline T1w & & & gap & Yes & 4 & $25.0 \%$ \\
\hline & & & gap & No & 12 & $75.0 \%$ \\
\hline None & 15 & $32.6 \%$ & & & & \\
\hline
\end{tabular}


Table 2. Cont

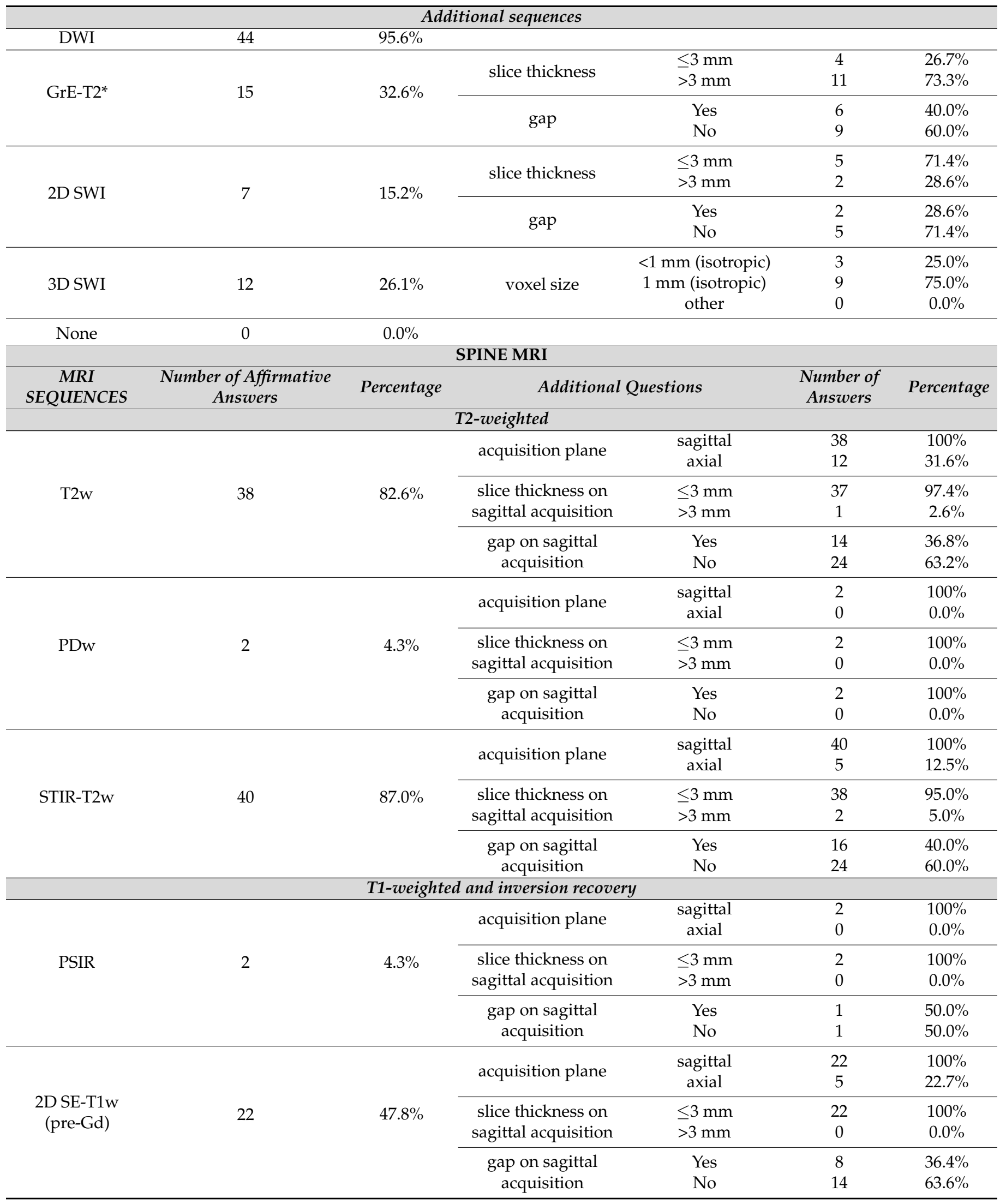


Table 2. Cont

\begin{tabular}{|c|c|c|c|c|c|c|}
\hline \multirow{3}{*}{$\begin{array}{l}\text { 3D GrE-T1w } \\
\text { (pre-Gd) }\end{array}$} & \multirow{3}{*}{2} & \multirow{3}{*}{$4.3 \%$} & acquisition plane & $\begin{array}{l}\text { sagittal } \\
\text { axial }\end{array}$ & $\begin{array}{l}2 \\
1 \\
\end{array}$ & $\begin{array}{l}100 \% \\
50.0 \% \\
\end{array}$ \\
\hline & & & $\begin{array}{c}\text { slice thickness on } \\
\text { sagittal acquisition }\end{array}$ & $\begin{array}{l}\leq 3 \mathrm{~mm} \\
>3 \mathrm{~mm}\end{array}$ & $\begin{array}{l}2 \\
0\end{array}$ & $\begin{array}{l}100 \% \\
0.0 \%\end{array}$ \\
\hline & & & $\begin{array}{l}\text { gap on sagittal } \\
\text { acquisition }\end{array}$ & $\begin{array}{l}\text { Yes } \\
\text { No }\end{array}$ & $\begin{array}{l}0 \\
2\end{array}$ & $\begin{array}{l}0.0 \% \\
100 \%\end{array}$ \\
\hline \multirow{4}{*}{$\begin{array}{l}\text { 2D SE-T1w } \\
\text { (post-Gd) }\end{array}$} & \multirow{4}{*}{36} & \multirow{4}{*}{$78.3 \%$} & acquisition plane & $\begin{array}{l}\text { sagittal } \\
\text { axial }\end{array}$ & $\begin{array}{c}36 \\
9\end{array}$ & $\begin{array}{l}100 \% \\
25.0 \%\end{array}$ \\
\hline & & & $\begin{array}{l}\text { slice thickness on } \\
\text { sagittal acquisition }\end{array}$ & $\begin{array}{l}\leq 3 \mathrm{~mm} \\
>3 \mathrm{~mm}\end{array}$ & $\begin{array}{c}33 \\
2\end{array}$ & $\begin{array}{c}91.6 \% \\
5.5 \%\end{array}$ \\
\hline & & & $\begin{array}{l}\text { gap on sagittal } \\
\text { acquisition }\end{array}$ & $\begin{array}{l}\text { Yes } \\
\text { No }\end{array}$ & $\begin{array}{l}13 \\
22\end{array}$ & $\begin{array}{l}36.1 \% \\
61.1 \%\end{array}$ \\
\hline & & & delay & $\begin{array}{l}\leq 5^{\prime} \\
>5^{\prime}\end{array}$ & $\begin{array}{l}17 \\
19\end{array}$ & $\begin{array}{l}47.2 \% \\
52.8 \%\end{array}$ \\
\hline \multirow{4}{*}{$\begin{array}{l}\text { 3D GrE-T1w } \\
\text { (post-Gd) }\end{array}$} & \multirow{4}{*}{4} & \multirow{4}{*}{$8.7 \%$} & acquisition plane & $\begin{array}{l}\text { sagittal } \\
\text { axial }\end{array}$ & $\begin{array}{l}4 \\
1\end{array}$ & $\begin{array}{l}100 \% \\
25.0 \%\end{array}$ \\
\hline & & & $\begin{array}{l}\text { slice thickness on } \\
\text { sagittal acquisition }\end{array}$ & $\begin{array}{l}\leq 3 \mathrm{~mm} \\
>3 \mathrm{~mm}\end{array}$ & $\begin{array}{l}3 \\
1\end{array}$ & $\begin{array}{l}75.0 \% \\
25.0 \% \\
\end{array}$ \\
\hline & & & $\begin{array}{l}\text { gap on sagittal } \\
\text { acquisition }\end{array}$ & $\begin{array}{l}\text { Yes } \\
\text { No }\end{array}$ & $\begin{array}{l}1 \\
3\end{array}$ & $\begin{array}{l}25.0 \% \\
75.0 \%\end{array}$ \\
\hline & & & delay & $\begin{array}{l}\leq 5^{\prime} \\
>5^{\prime}\end{array}$ & $\begin{array}{l}0 \\
4\end{array}$ & $\begin{array}{l}0.0 \% \\
100 \%\end{array}$ \\
\hline
\end{tabular}

SE = spin-echo; GrE = gradient-echo; FLAIR = fluid attenuated inversion recovery; DIR = double inversion recovery; PSIR = phase sensitive inversion recovery; Fat-Sat = fat saturated; DWI = diffusion weighted imaging; SWI = susceptibility weighted imaging; $\mathrm{PD}=$ proton density; STIR = short tau inversion recovery; $\mathrm{Gd}$ = gadolinium .

\subsection{Stratification By Work-Related Environment}

When stratifying results on the basis of the respondents' workplace, we found that the acquisition of the 3D FLAIR sequence was substantially comparable (private clinics: 8/9, 88.9\%; hospitals: 20/23, 87.0\%; university hospitals: $12 / 14,85.7 \%$ ). Regarding brain sequences acquired after Gadolinium administration, the 2D SE-T1w was the preferred choice in hospitals $(13 / 21,61.9 \%)$ and university hospitals $(8 / 14,57.1 \%)$, while 3D GrE-T1w was the most acquired sequence in private clinics $(6 / 9,66.7 \%)$. When a 2D SE-T1w was acquired, it was often obtained with a slice thickness superior to $3 \mathrm{~mm}(16 / 26,61.5 \%)$, mostly in private clinical centers $(4 / 5,80.0 \%)$ and hospitals $(8 / 13,61.5 \%)$, but also in university hospitals $(4 / 8,50.0 \%)$.

\subsection{Stratification by Years of Experience}

When stratifying data by years of experience, we found that all NRs with less than 10 years of experience usually acquired a 3D FLAIR sequence $(22 / 22,100.0 \%)$, whereas this percentage was reduced to $77.3 \%(17 / 22)$ when evaluating results obtained from NRs with more than 10 years of experience. Regarding the Gadolinium contrast-enhanced sequences, we found that the 2D SE-T1w was the most performed sequence by young NRs $(13 / 18,72.2 \%)$, while the subgroup of more experienced ones acquired both 3D-GrE-T1w and 2D SE-T1w in less than 50\% of the cases $(9 / 22,40.9 \%)$. Notably, the 2D SE-T1w was performed with a slice thickness superior to $3 \mathrm{~mm}$ in almost all cases by older NRs $(8 / 9$, $88.9 \%$ ). Finally, the report structure also proved to be slightly different between less and more experienced NRs, with a structured report being made by young NRs in half of the cases $(4 / 8,50.0 \%)$. 


\section{FOLLOW-UP}

\section{Brain MRI}

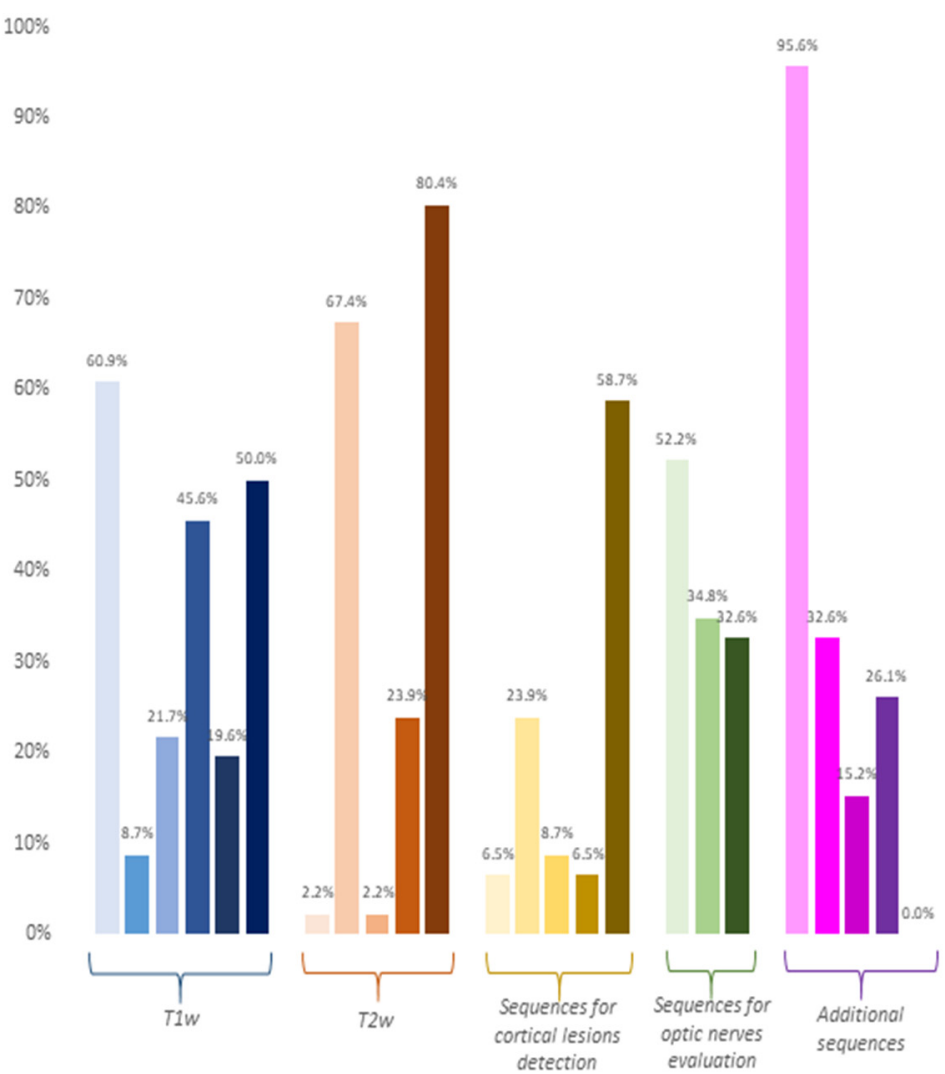

2D SE-T1w (pre-Gd)

- 3D SE-T1w (pre-Gd)

n 3D GrE-T1w (pre-Gd)

- 2D SE-T1w (post-Gd)

- 3D SE-T1w (post-Gd)

- 3D GrE-T1w (post-Gd)

PD/T2W

घ $2 \mathrm{DT} 2 \mathrm{w}$

$=3 \mathrm{DT} 2 \mathrm{w}$

- 2D FLAIR

- 3D FLAIR

2D DIR

SDDIR

2DPSIR

a 3D PSIR

- None

STIR-T2w

a Post-Gd Fat-Sat T1w

- None

ㅁWI

- GrE-T2* $w$

- 2DSWI

-3D SWI

a None

Spine MRI

$100 \%$
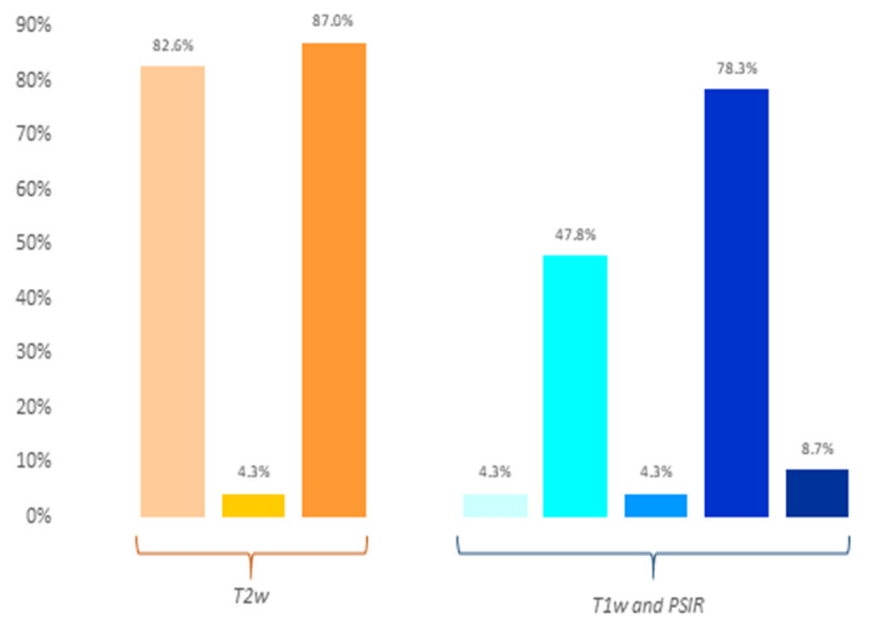

$\because \mathrm{T} 2 \mathrm{w}$

" $\mathrm{PDW}$

๓STR-T2W

PSIR

2D SE-T1w (pre-Gd)

a 3D GrE-T1w (pre-Gd)

- 2D SE-T1w (post-Gd)

- $3 D$ GrE-T1w (post-Gd)

Figure 3. Sequences included in the follow-up brain and spine MRI protocols. Data are show as percentages. SE = spin-echo; $\mathrm{GrE}$ = gradient-echo; FLAIR = fluid attenuated inversion recovery; DIR = double inversion recovery; PSIR = phase sensitive inversion recovery; Fat-Sat = fat saturated; DWI = diffusion weighted imaging; SWI = susceptibility weighted imaging; $\mathrm{PD}=$ proton density; STIR = short tau inversion recovery; $\mathrm{Gd}=$ gadolinium . 


\subsection{Report Structure}

Regarding the report structure, most of the participants $(38 / 46,82.6 \%)$ routinely preferred a descriptive report over a standardized one. In most of the cases, report drafting required 15 to $30 \mathrm{~min}$ per patient $(31 / 46,67.4 \%)$. Only a few NRs $(14 / 46,30.4 \%)$ clearly referred to the 2017 revision of the McDonald criteria for MS diagnosis in their report. In a relatively large number of cases, NRs specified the MRI protocol used $(41 / 46,89.1 \%)$, the anatomical areas covered $(35 / 46,76.1 \%)$, and the contrast agent type and dose $(34 / 46$, $73.9 \%)$. On the other hand, in less than half of the cases $(19 / 46,41.3 \%)$, the magnetic field intensity was included in the report, and slice thickness was only rarely declared (5/46, $10.9 \%$ ). Most of participants included in their report information about lesions number $(31 / 46,67.4 \%)$, as a range and not as an exact number, as well as the precise number of contrast enhancing lesions. Regarding lesion localization, almost all participants routinely specify the lesion location $(45 / 46,97.8 \%)$, focusing on only typical MS locations in most cases $(25 / 45,55.6 \%)$ rather than specifying the specific anatomic region affected $(20 / 45$, $44.4 \%)$. Less than $20 \%$ of the participants $(8 / 46,17.4 \%$ ) clearly reported the presence of optic nerves involvement, while information about the presence of atrophy (via qualitative evaluation, $39 / 46,84.8 \%)$, black holes $(38 / 46,82.6 \%)$, and cortical lesions number $(37 / 46$, $80.4 \%$ ) were frequently included. Finally, we observed a substantial correspondence between information provided at baseline and follow-up reports, with information about new lesions (43/46. 93.5\%) and their volume increase (42/46, 91.3\%) on T2w sequences also provided at follow-up.

\subsection{Future Directions}

Lastly, the participants were asked to select which MS emerging physiopathological marker they believed to be ready for clinical use. Most of them indicated the central vein sign and the evaluation of brain atrophy via a quantitative assessment (in both cases, $24 / 46,52.2 \%$ ) as the two most plausible future diagnostic markers of the disease, while the evaluation of the "slowly expanding lesions" was judged relatively far from a possible use in a clinical setting $(6 / 46,13 \%)$. Nine out of 46 NRs $(19.6 \%)$ claimed that none of the proposed signs were ready to be translated into clinical practice, mostly because they felt there was a lack of proper preparation to recognize those markers $(6 / 9,66.7 \%)$ or believed technical limitations would hamper the acquisition of specific sequences required for biomarkers assessment $(4 / 9,44.4 \%)$.

\section{Discussion}

The central role of MRI in the evaluation of MS patients is unquestionable [11]. Over the years, MRI has become one of the most important diagnostic tools available to NRs and clinicians, also providing relevant insights about the pathophysiology of damage in MS and offering new possibilities to monitor disease progression and treatment response [12]. In light of the crucial role of MRI in clinical settings [13], expert guidelines have been produced to ensure that MRI protocols for evaluations of MS patients would include sequences adequate for the application of the ever-evolving MS diagnostic criteria and longitudinal monitoring of disease activity. Specifically, the MAGNIMS committee identifies mandatory and optional sequences. Among the mandatory sequences, a particular emphasis is placed on the use of FLAIR-T2w sequences and post-contrast T1w sequences [8]. The results of this study show that although the MS community is somehow close to a standardization of MRI protocols, there is still room for a certain degree of variability in clinical practice. For instance, although most of the participants demonstrated an overall good adherence to the recommendations about post-gadolinium sequences, almost $10 \%$ of the participants did not follow the MAGNIMS recommendations, only acquiring 2D sequences with a slice thickness superior to $3 \mathrm{~mm}$. A possible explanation could be the need for reducing acquisition times, although the diagnostic efficacy of such an approach is obviously debatable. In this light, an additional nonadherence to the current guidelines, perhaps even more crucial than the previous one, was that about $30 \%$ of NR claimed to acquire 
the post-contrast $\mathrm{T} 1 \mathrm{w}$ sequences within $5 \mathrm{~min}$ of the contrast administration. This point is crucial, given that it is well established that lesions peak enhancement occurs $10 \mathrm{~min}$ after contrast administration $[10,14]$. Changes regarding the timing of post-gadolinium T1w acquisition could affect the diagnostic workflow of these patients, given that contrast enhancement allows for determination of DIT at diagnosis [4]. Finally, and still related to gadolinium administration issues, the results of this study suggest a usage of contrast administration not in line with the current guidelines, both at baseline (where almost 10\% of participants declared to not routinely acquire post-Gd sequence in cases of suspected MS) and especially at follow-up. In particular, we found a similar percentage of NRs routinely administering a contrast agent both at baseline and follow-up examinations. A possible explanation for this phenomenon could be researched in a historical heritage, as for years, contrast administration was thought to be the only way to demonstrate disease activity and progression, even using double or triple doses [15-17]. Nevertheless, it is now well established that disease progression can be assessed by evaluating different MRI parameters, although unfortunately it appears that such information does not translate into a reduced request for and execution of post gadolinium sequences. Beyond the relative utility of contrast agent administration in follow-up studies, recent concerns about brain gadolinium deposition [18-20] represent an additional reason to limit the use of gadolinium-based agents to specific cases, although the clinical impact of gadolinium deposition is far from being completely understood [21-23]. As per the opportunity to evaluate the presence of CL, not only is cortical involvement considered as typical by the most recent MS diagnostic criteria, but numerous studies have shown that its presence and number is correlated with both motor and cognitive disability in MS patients [24-28] since the early phases of the disease [29]. Accordingly, about $80 \%$ of NRs routinely evaluated the presence of $C L$ in MS baseline evaluation, via the application of dedicated sequences in half of the cases. Among these, DIR was the most widely applied in our sample, in line with the increased attention gained by this sequence in recent years [30,31]. It has to be noted that in a very small percentage of cases (less than 5\%), NRs answered that they routinely work on a scanner with a field strength inferior to $1.5 \mathrm{~T}$, acquiring only standard clinical sequences, such as 2D SE-T1w, 2D T2w, and FLAIR sequences, thus further reducing the possibility to evaluate such lesions in this small subgroup. Regarding the study report, in our sample, NRs preferred a descriptive report over a standardized one. As per other districts and conditions [32-34], the use of a standardized structured report in MS is strongly warranted [35,36], given the intrinsic potential of such an approach to improve diagnostic accuracy and reproducibility over time, by solving problems such as ambiguity and lack of completeness that can occur with conventional narrative reports, with an obvious and direct impact on the quality of care. In this light, it was interesting to note that a relatively low percentage of participants usually mention the 2017 revision of the McDonald Criteria [4] in their report. Although it emerged that NRs in almost all cases refer in their report to typical locations needed to achieve DIS, it would be advisable to clearly state the current diagnostic criteria in the report, in line with the MAGNIMS consensus guidelines suggesting that the report must always contain a conclusion to communicate the radiological interpretation in relation to the clinical problem. Finally, participants were asked which, if any, of the emerging imaging markers was deemed to be included in the near future as part of the neuroradiological evaluation of MS in clinical practice. Among all of the imaging biomarkers, more than half of the NRs identified the central vein sign as a promising and useful marker for MS diagnosis, in line with research studies suggesting its application to increase diagnostic sensitivity and specificity [37-40], followed by a quantitative assessment of cerebral atrophy. As per this latter biomarker, although measures of brain atrophy are valuable markers of ongoing neurodegeneration in MS [41,42], and an atrophy cut-off able to differentiate pathological from physiological atrophy accrual has been identified [43], we must not forget that the determination of meaningful atrophy in the single-subject is still beyond our reach. Interestingly, less confidence in diagnostic sensitivity and specificity was reserved to other neuroradiological biomarkers such as the "slow-expanding lesions", 
probably due to methodological concerns. The main limitation of this study is represented by the small cohort of NRs involved. Indeed, although the survey reached a wide range of professional figures of various years of experience and Work-Related Environment, the overall numerosity is unfortunately too low to make assumptions about a more profound generalization of these findings. For this reason, future similar studies are warranted, reaching a larger number of international participants, to confirm the findings reported here. Although characterized by this limitation, in conclusion, this work showed that even if recommendations about standardized MRI protocols are present, in clinical practice, a relatively large degree of heterogeneity is still present regarding the neuroradiological evaluation of MS patients. Further work to overcome this issue is mandatory in the MS community to reduce differences among centers with the final aim to increase the quality of patients' care in this condition.

Supplementary Materials: The followings are available online at https:/ / www.mdpi.com/article/ 10.3390/diagnostics11081310/s1.

Author Contributions: Conceptualization: G.P., M.P. (Maria Petracca), M.T. (Mario Tortora), M.T. (Mario Tranfa); methodology: A.C.D., M.P. (Maria Petracca), S.C.; software: A.C.D., M.P. (Maria Petracca); formal analysis, M.T. (Mario Tortora), M.T. (Mario Tranfa); investigation: All Authors; resources, A.B. (Arturo Brunetti), A.E.; data curation, S.C.; writing-original draft preparation: M.T. (Mario Tortora), M.T. (Mario Tranfa), A.C.D.; writing-review and editing, M.T. (Mario Tortora), M.T. (Mario Tranfa), A.C.D., G.P., M.P. (Maria Petracca), A.B. (Alessandro Bozzao), F.C., A.C., M.C., A.F., M.L., R.M., M.M., M.P. (Michele Porcu), L.R., A.T., L.S., A.B. (Arturo Brunetti), S.C., A.E.; supervision: A.B. (Arturo Brunetti), A.E.; project administration, S.C., A.E. All authors have read and agreed to the published version of the manuscript.

Funding: S.C. received speaker fees from Sanofi and Amicus Therapeutics and a Research Grant from FISM (Fondazione Italiana Sclerosi Multipla).

Institutional Review Board Statement: Not applicable.

Informed Consent Statement: Not applicable.

Data Availability Statement: Data will be made available upon reasonable request to the corresponding author.

Acknowledgments: The Authors would like to thank all the participants to this study, as well as Antonio Armentano, Claudio Colonnese, Stefania Graziuso, Antonio Lopez, Giovanni Moggio, Maria Pia Pappalardo, Teresa Popolizio, and Rossana Senese for their precious assistance in disseminating the survey.

Conflicts of Interest: The authors declare no conflict of interest.

\section{References}

1. Compston, A.; Coles, A. Multiple sclerosis. Lancet 2008, 372, 1502-1517. [CrossRef]

2. Lublin, F.D.; Reingold, S.C.; Cohen, J.A.; Cutter, G.R.; Sørensen, P.S.; Thompson, A.J.; Wolinsky, J.S.; Balcer, L.J.; Banwell, B.; Barkhof, F.; et al. Defining the clinical course of multiple sclerosis: The 2013 revisions. Neurology 2014, 83, 278-286. [CrossRef]

3. Gelfand, J.M. Multiple sclerosis: Diagnosis, differential diagnosis, and clinical presentation. In Handbook of Clinical Neurology; Elsevier B.V.: Amsterdam, The Netherlands, 2014; Volume 122, pp. 269-290.

4. Thompson, A.J.; Banwell, B.L.; Barkhof, F.; Carroll, W.M.; Coetzee, T.; Comi, G.; Correale, J.; Fazekas, F.; Filippi, M.; Freedman, M.S.; et al. Diagnosis of multiple sclerosis: 2017 revisions of the McDonald criteria. Lancet Neurol. 2018, 17, 162-173. [CrossRef]

5. Aliaga, E.S.; Barkhof, F. MRI mimics of multiple sclerosis. In Handbook of Clinical Neurology; Elsevier B.V.: Amsterdam, The Netherlands, 2014; Volume 122, pp. 291-316.

6. Wattjes, M.P.; Steenwijk, M.D.; Stangel, M. MRI in the Diagnosis and Monitoring of Multiple Sclerosis: An Update. Clin. Neuroradiol. 2015, 25 (Suppl. S2), 157-165. [CrossRef] [PubMed]

7. Filippi, M.; Preziosa, P.; Banwell, B.L.; Barkhof, F.; Ciccarelli, O.; De Stefano, N.; Geurts, J.J.G.; Paul, F.; Reich, D.S.; Toosy, A.T.; et al. Assessment of lesions on magnetic resonance imaging in multiple sclerosis: Practical guidelines. Brain J. Neurol. 2019, 142, 1858-1875. [CrossRef] [PubMed]

8. Traboulsee, A.; Simon, J.H.; Stone, L.; Fisher, E.; Jones, D.E.; Malhotra, A.; Newsome, S.D.; Oh, J.; Reich, D.S.; Richert, N.; et al. Revised Recommendations of the Consortium of MS Centers Task Force for a Standardized MRI Protocol and Clinical Guidelines for the Diagnosis and Follow-Up of Multiple Sclerosis. AJNR Am. J. Neuroradiol. 2016, 37, 394-401. [CrossRef] [PubMed] 
9. Filippi, M.; Rocca, M.A.; Ciccarelli, O.; De Stefano, N.; Rammohan, K.; Khan, O.; Radue, E.-W.; Ford, C.; Halper, J.; Li, D. MRI criteria for the diagnosis of multiple sclerosis: MAGNIMS consensus guidelines. Lancet Neurol. 2016, 15, 292-303. [CrossRef]

10. Rovira, À.; Wattjes, M.P.; Tintoré, M.; Tur, C.; Yousry, T.A.; Sormani, M.P.; De Stefano, N.; Filippi, M.; Auger, C.; Rocca, M.A.; et al. Evidence-based guidelines: MAGNIMS consensus guidelines on the use of MRI in multiple sclerosis-clinical implementation in the diagnostic process. Nat. Rev. Neurol. 2015, 11, 471-482. [CrossRef]

11. Curley, M.; Josey, L.; Lucas, R.; McMichael, T.; Pender, M.P.; Ponsonby, A.-L.; Taylor, B.; Valery, P.; van der Mei, I.; Williams, D. Adherence to MRI protocol consensus guidelines in multiple sclerosis: An Australian multi-centre study. J. Med. Imaging Radiat. Oncol. 2012, 56, 594-598. [CrossRef]

12. Gasperini, C.; Prosperini, L.; Tintoré, M.; Sormani, M.P.; Filippi, M.; Rio, J.; Palace, J.; Rocca, M.A.; Ciccarelli, O.; Barkhof, F.; et al. Unraveling treatment response in multiple sclerosis: A clinical and MRI challenge. Neurology 2019, 92, 180-192. [CrossRef]

13. Vola, E.A.; Petracca, M.; Cocozza, S.; De Angelis, M.; Carotenuto, A.; Pontillo, G.; Morra, V.B.; Tedeschi, E.; Lanzillo, R. Possible progressive multifocal leukoencephalopathy and active multiple sclerosis under dimethyl fumarate: The central role of MRI in informing therapeutic decisions. BMC Neurol. 2021, 21, 146. [CrossRef]

14. Rovaris, M.; Filippi, M. Contrast enhancement and the acute lesion in multiple sclerosis. Neuroimaging Clin. N. Am. 2000, 10, 705-716. [PubMed]

15. Filippi, M.; Yousry, T.; Campi, A.; Kandziora, C.; Colombo, B.; Voltz, R.; Martinelli, V.; Spuler, S.; Bressi, S.; Scotti, G.; et al. Comparison of triple dose versus standard dose gadolinium-DTPA for detection of MRI enhancing lesions in patients with MS. Neurology 1996, 46, 379-384. [CrossRef] [PubMed]

16. van Waesberghe, J.H.; Castelijns, J.A.; Roser, W.; Silver, N.; Yousry, T.; Nijeholt, G.J.L.à.; Adèr, H.J.; Uitdehaag, B.M.; Radue, E.W.; Polman, C.H.; et al. Single-dose gadolinium with magnetization transfer versus triple-dose gadolinium in the MR detection of multiple sclerosis lesions. Am. J. Neuroradiol. 1997, 18, 1279-1285.

17. Wolansky, L.J.; Finden, S.G.; Chang, R.; Conigliari, M.; Lee, H.J.; Shaderowsky, P.D.; Cook, S.D. Gadoteridol in multiple sclerosis patients. A comparison of single and triple dose with immediate vs. delayed imaging. Clin. Imaging 1998, 22, 385-392. [CrossRef]

18. Kanda, T.; Ishii, K.; Kawaguchi, H.; Kitajima, K.; Takenaka, D. High signal intensity in the dentate nucleus and globus pallidus on unenhanced T1-weighted MR images: Relationship with increasing cumulative dose of a gadolinium-based contrast material. Radiology 2014, 270, 834-841. [CrossRef] [PubMed]

19. McDonald, R.J.; McDonald, J.S.; Kallmes, D.F.; Jentoft, M.E.; Murray, D.L.; Thielen, K.R.; Williamson, E.E.; Eckel, L.J. Intracranial Gadolinium Deposition after Contrast-enhanced MR Imaging. Radiology 2015, 275, 772-782. [CrossRef] [PubMed]

20. Tedeschi, E.; Palma, G.; Canna, A.; Cocozza, S.; Russo, C.; Borrelli, P.; Lanzillo, R.; Angelini, V.; Postiglione, E.; Morra, V.B.; et al. In vivo dentate nucleus MRI relaxometry correlates with previous administration of Gadolinium-based contrast agents. Eur. Radiol. 2016, 26, 4577-4584. [CrossRef] [PubMed]

21. Cocozza, S.; Pontillo, G.; Lanzillo, R.; Russo, C.; Petracca, M.; Stasi, M.D.; Paolella, C.; Vola, E.A.; Criscuolo, C.; Moccia, M.; et al. MRI features suggestive of gadolinium retention do not correlate with Expanded Disability Status Scale worsening in Multiple Sclerosis. Neuroradiology 2019, 61, 155-162. [CrossRef] [PubMed]

22. Forslin, Y.; Shams, S.; Hashim, F.; Aspelin, P.; Bergendal, G.; Martola, J.; Fredrikson, S.; Kristoffersen-Wiberg, M.; Granberg, T. Retention of Gadolinium-Based Contrast Agents in Multiple Sclerosis: Retrospective Analysis of an 18-Year Longitudinal Study. AJNR Am. J. Neuroradiol. 2017, 38, 1311-1316. [CrossRef] [PubMed]

23. Mallio, C.A.; Rovira, À.; Parizel, P.M.; Quattrocchi, C.C. Exposure to gadolinium and neurotoxicity: Current status of preclinical and clinical studies. Neuroradiology 2020, 62, 925-934. [CrossRef]

24. Calabrese, M.; Agosta, F.; Rinaldi, F.; Mattisi, I.; Grossi, P.; Favaretto, A.; Atzori, M.; Bernardi, V.; Barachino, L.; Rinaldi, L.; et al. Cortical lesions and atrophy associated with cognitive impairment in relapsing-remitting multiple sclerosis. Arch. Neurol. 2009, 66, 1144-1150. [CrossRef]

25. Curti, E.; Graziuso, S.; Tsantes, E.; Crisi, G.; Granella, F. Correlation between cortical lesions and cognitive impairment in multiple sclerosis. Brain Behav. 2018, 8, e00955. [CrossRef]

26. Harrison, D.M.; Roy, S.; Oh, J.; Izbudak, I.; Pham, D.; Courtney, S.; Caffo, B.; Jones, C.K.; van Zijl, P.; Calabresi, P.A. Association of Cortical Lesion Burden on 7-T Magnetic Resonance Imaging With Cognition and Disability in Multiple Sclerosis. JAMA Neurol. 2015, 72, 1004-1012. [CrossRef]

27. Pirko, I.; Lucchinetti, C.F.; Sriram, S.; Bakshi, R. Gray matter involvement in multiple sclerosis. Neurology 2007, 68, 634-642. [CrossRef]

28. Roosendaal, S.D.; Moraal, B.; Pouwels, P.J.; Vrenken, H.; Castelijns, J.A.; Barkhof, F.; Geurts, J.J.G. Accumulation of cortical lesions in MS: Relation with cognitive impairment. Mult. Scler. 2009, 15, 708-714. [CrossRef]

29. Calabrese, M.; De Stefano, N.; Atzori, M.; Bernardi, V.; Mattisi, I.; Barachino, L.; Morra, A.; Rinaldi, L.; Romualdi, C.; Perini, P.; et al. Detection of cortical inflammatory lesions by double inversion recovery magnetic resonance imaging in patients with multiple sclerosis. Arch. Neurol. 2007, 64, 1416-1422. [CrossRef] [PubMed]

30. Calabrese, M.; Battaglini, M.; Giorgio, A.; Atzori, M.; Bernardi, V.; Mattisi, I.; Gallo, P.; De Stefano, N. Imaging distribution and frequency of cortical lesions in patients with multiple sclerosis. Neurology 2010, 75, 1234-1240. [CrossRef] [PubMed]

31. Geurts, J.J.; Pouwels, P.J.; Uitdehaag, B.M.; Polman, C.H.; Barkhof, F.; Castelijns, J.A. Intracortical lesions in multiple sclerosis: Improved detection with 3D double inversion-recovery MR imaging. Radiology 2005, 236, 254-260. [CrossRef] [PubMed] 
32. Brown, P.J.; Rossington, H.; Taylor, J.; Lambregts, D.M.J.; Morris, E.; West, N.P.; Quirke, P.; Tolan, D.; on behalf of the YCR BCIP Study Group. Standardised reports with a template format are superior to free text reports: The case for rectal cancer reporting in clinical practice. Eur. Radiol. 2019, 29, 5121-5128. [CrossRef] [PubMed]

33. Stanzione, A.; Boccadifuoco, F.; Cuocolo, R.; Romeo, V.; Mainenti, P.P.; Brunetti, A.; Maurea, S. State of the art in abdominal MRI structured reporting: A review. Abdom. Radiol. 2021, 46, 1218-1228. [CrossRef] [PubMed]

34. Wibmer, A.; Vargas, H.A.; Sosa, R.; Zheng, J.; Moskowitz, C.; Hricak, H. Value of a standardized lexicon for reporting levels of diagnostic certainty in prostate MRI. AJR Am. J. Roentgenol. 2014, 203, W651-W657. [CrossRef] [PubMed]

35. Alessandrino, F.; Pichiecchio, A.; Mallucci, G.; Ghione, E.; Romani, A.; Bergamaschi, R.; Bastianello, S. Do MRI Structured Reports for Multiple Sclerosis Contain Adequate Information for Clinical Decision Making? AJR Am. J. Roentgenol. 2018, $210,24-29$. [CrossRef]

36. Lee, J.K.; Bermel, R.; Bullen, J.; Ruggieri, P.; Jones, S.E. Structured Reporting in Multiple Sclerosis Reduces Interpretation Time. Acad. Radiol. 2020. [CrossRef]

37. Sinnecker, T.; Clarke, M.A.; Meier, D.; Enzinger, C.; Calabrese, M.; De Stefano, N.; Pitiot, A.; Giorgio, A.; Schoonheim, M.M.; Paul, F.; et al. Evaluation of the Central Vein Sign as a Diagnostic Imaging Biomarker in Multiple Sclerosis. JAMA Neurol. 2019, 76, 1446-1456. [CrossRef] [PubMed]

38. Mistry, N.; Abdel-Fahim, R.; Samaraweera, A.; Mougin, O.; Tallantyre, E.; Tench, C.; Jaspan, T.; Morris, P.; Morgan, P.S.; Evangelou, $\mathrm{N}$. Imaging central veins in brain lesions with 3-T T2*-weighted magnetic resonance imaging differentiates multiple sclerosis from microangiopathic brain lesions. Mult. Scler. 2016, 22, 1289-1296. [CrossRef] [PubMed]

39. Maggi, P.; Absinta, M.; Grammatico, M.; Vuolo, L.; Emmi, G.; Carlucci, G.; Spagni, G.; Barilaro, A.; Repice, A.M.; Emmi, L.; et al. Central vein sign differentiates Multiple Sclerosis from central nervous system inflammatory vasculopathies. Ann. Neurol. 2018, 83, 283-294. [CrossRef] [PubMed]

40. Guisset, F.; Lolli, V.; Bugli, C.; Perrotta, G.; Absil, J.; Dachy, B.; Pot, C.; Théaudin, M.; Pasi, M.; van Pesch, V.; et al. The central vein sign in multiple sclerosis patients with vascular comorbidities. Mult. Scler. 2021, 27, 1057-1065. [CrossRef] [PubMed]

41. Inglese, M.; Petracca, M. MRI in multiple sclerosis: Clinical and research update. Curr. Opin. Neurol. 2018, 31, 249-255. [CrossRef] [PubMed]

42. Pontillo, G.; Cocozza, S.; Di Stasi, M.; Carotenuto, A.; Paolella, C.; Cipullo, M.B.; Perillo, T.; Vola, E.A.; Russo, C.; Masullo, M.; et al. 2D linear measures of ventricular enlargement may be relevant markers of brain atrophy and long-term disability progression in multiple sclerosis. Eur. Radiol. 2020, 30, 3813-3822. [CrossRef] [PubMed]

43. De Stefano, N.; Stromillo, M.L.; Giorgio, A.; Bartolozzi, M.L.; Battaglini, M.; Baldini, M.; Portaccio, E.; Amato, M.P.; Sormani, M.P. Establishing pathological cut-offs of brain atrophy rates in multiple sclerosis. J. Neurol. Neurosurg. Psychiatry 2016, 87, 93-99. [CrossRef] [PubMed] 